PROCEEDINGS OF THE

AMERICAN MATHEMATICAL SOCIETY

Volume 134, Number 5, Pages 1289-1298

S 0002-9939(05)08171-2

Article electronically published on October 18, 2005

\title{
THE HYPERSURFACE $x+x^{2} y+z^{2}+t^{3}=0$ OVER A FIELD OF ARBITRARY CHARACTERISTIC
}

\author{
ANTHONY J. CRACHIOLA \\ (Communicated by Bernd Ulrich) \\ To Professor Leonid Makar-Limanov on the occasion of his sixtieth birthday

\begin{abstract}
We develop techniques for computing the AK invariant of domains with arbitrary characteristic. As an example, we show that for any field $\mathbf{k}$ the ring $\mathbf{k}[X, Y, Z, T] /\left(X+X^{2} Y+Z^{2}+T^{3}\right)$ is not isomorphic to a polynomial ring over $\mathbf{k}$.
\end{abstract}

\section{INTRODUCTION}

All rings in this paper are commutative with identity. Throughout this paper, let $\mathbf{k}$ denote a field of arbitrary characteristic, and let $\mathbf{k}^{*}=\mathbf{k} \backslash 0$. For a ring $A$, let $A^{[n]}$ denote the polynomial ring in $n$ indeterminates over $A$. Let $\mathbf{C}$ denote the complex number field.

The AK invariant was introduced by Leonid Makar-Limanov [M1] to show that Peter Russell's [KR threefold

$$
x+x^{2} y+z^{2}+t^{3}=0
$$

over $\mathbf{C}$ is not isomorphic to $\mathbf{C}^{3}$. The invariant was defined in that context as the intersection of the kernels of all locally nilpotent derivations on the coordinate ring of a variety. Since this first application, the AK invariant has been applied by several people (for instance $[\mathrm{BM}, \overline{\mathrm{Du}}, \overline{\mathrm{KKMR}}]$ ), mainly in the realm of affine algebraic geometry. To the author's knowledge, this work has all been conducted under the restriction of zero characteristic. Superficially, this is reasonable, since derivations do not behave as nicely on rings with prime characteristic $p$. The kernel of such a derivation does not convey the appropriate information in this setting, since it will always contain the $p$ th power of every element.

Nevertheless, the apparent advantage to restricting the characteristic may be only a matter of perception. In the zero characteristic situation, locally nilpotent derivations on the coordinate ring correspond with algebraic additive group actions on the variety, and, unlike locally nilpotent derivations, these actions maintain their attractive properties in the prime characteristic setting. While the zero characteristic setting may seem more topologically natural or intuitive, we sometimes find that only the algebraic structure is required in obtaining desired results. As one

Received by the editors August 25, 2004 and, in revised form, December 26, 2004.

2000 Mathematics Subject Classification. Primary 13A50; Secondary 14J30, 14R20.

Key words and phrases. AK invariant, additive group action, locally finite iterative higher derivation.

(C)2005 American Mathematical Society
Reverts to public domain 28 years from publication 1289 
example, after Rudolf Rentschler $\left[\mathrm{R}\right.$ provided a description of algebraic $\mathbf{C}^{+}$-actions on $\mathbf{C}^{2}$, Masayoshi Miyanishi [Mi] demonstrated that Rentschler's theorem extends naturally without any technical difficulty to an affine plane of arbitrary characteristic. As another example, after Takao Fujita, Miyanishi and Tohru Sugie [F, MS] affirmatively solved the Zariski cancellation problem for the affine plane $\mathbf{C}^{2}$, Russell $[\mathrm{Ru}$ gave a simplified treatment of their proof which erased the characteristic zero restriction.

The purpose of the present paper is to place the AK invariant in a characteristicfree environment. We provide the definition and basic ideas, and we demonstrate computational techniques, closely following earlier treatments due to Harm Derksen, Ofer Hadas, and Makar-Limanov [D, DHM, M1, which gain no simplification from the characteristic zero assumption. As an illustration, we compute the AK invariant for $x+x^{2} y+z^{2}+t^{3}=0$ over any field $\mathbf{k}$ and obtain the same result which was originally found over C. Similar efforts have been successfully applied toward a generalization of the Zariski cancellation problem [CM].

\section{Methods}

Exponential maps, the AK invariant, and locally finite iterative higher derivations. Let $A$ be a k-algebra. Suppose $\varphi: A \rightarrow A^{[1]}$ is a k-algebra homomorphism. We write $\varphi=\varphi_{U}: A \rightarrow A[U]$ if we wish to emphasize an indeterminate $U$. We say that $\varphi$ is an exponential map on $A$ if it satisfies the following two additional properties:

(i) $\varepsilon_{0} \varphi_{U}$ is the identity on $A$, where $\varepsilon_{0}: A[U] \rightarrow A$ is evaluation at $U=0$.

(ii) $\varphi_{S} \varphi_{U}=\varphi_{S+U}$, where $\varphi_{S}$ is extended by $\varphi_{S}(U)=U$ to a homomorphism $A[U] \rightarrow A[S, U]$.

(When $A$ is the coordinate ring of an affine variety $\operatorname{Spec}(A)$ over $\mathbf{k}$, the exponential maps on $A$ correspond to algebraic actions of the additive group $\mathbf{k}^{+}$on $\operatorname{Spec}(A)[\mathrm{E}$, §9.5].) Define

$$
A^{\varphi}=\{a \in A \mid \varphi(a)=a\}
$$

a subalgebra of $A$ called the ring of $\varphi$-invariants. Let $\operatorname{EXP}(A)$ denote the set of all exponential maps on $A$. We define the $A K$ invariant, or ring of absolute constants of $A$, as

$$
\operatorname{AK}(A)=\bigcap_{\varphi \in \operatorname{EXP}(A)} A^{\varphi} .
$$

This is a subalgebra of $A$ which is preserved by isomorphism. Indeed, any isomorphism $f: A \rightarrow B$ of $\mathbf{k}$-algebras restricts to an isomorphism $f: \operatorname{AK}(A) \rightarrow \operatorname{AK}(B)$. To understand this, observe that if $\varphi \in \operatorname{EXP}(A)$, then $f \varphi f^{-1} \in \operatorname{EXP}(B)$. Remark that $\operatorname{AK}(A)=A$ if and only if the only exponential map on $A$ is the standard inclusion $\varphi(a)=a$ for all $a \in A$.

Example 2.1. By considering exponential maps of the form $\varphi_{i}\left(X_{j}\right)=X_{j}+\delta_{i j} U$, where $\delta_{i j}$ is the Kronecker delta, one can see that $\operatorname{AK}\left(\mathbf{k}^{[n]}\right)=\mathbf{k}$ for each natural number $n$. When $n=1$, this characterizes $\mathbf{k}^{[1]}$ (see Lemma 2.4). However, if $A$ is a domain with transcendence degree $n \geq 2$ over $\mathbf{k}$, then $\operatorname{AK}(A)=\mathbf{k}$ does not imply that $A \cong \mathbf{k}^{[n]}[\mathrm{BM}$.

It is often helpful to view a given $\varphi \in \operatorname{EXP}(A)$ as a sequence in the following way. For each $a \in A$ and each natural number $n$, let $D^{n}(a)$ denote the $U^{n}$-coefficient of 
$\varphi(a)$. Let $D=\left\{D^{0}, D^{1}, D^{2}, \ldots\right\}$. To say that $\varphi$ is a $\mathbf{k}$-algebra homomorphism is equivalent to saying that the sequence $\left\{D^{i}(a)\right\}$ has finitely many nonzero elements for each $a \in A$, that $D^{n}: A \rightarrow A$ is $\mathbf{k}$-linear for each natural number $n$, and that the Leibniz rule

$$
D^{n}(a b)=\sum_{i+j=n} D^{i}(a) D^{j}(b)
$$

holds for all natural numbers $n$ and all $a, b \in A$. The above properties (i) and (ii) of the exponential map $\varphi$ translate into the following properties of $D$ :

(i') $D^{0}$ is the identity map.

(ii') (iterative property) For all natural numbers $i, j$,

$$
D^{i} D^{j}=\left(\begin{array}{c}
i+j \\
i
\end{array}\right) D^{i+j}
$$

Due to all of these properties, the collection $D$ is called a locally finite iterative higher derivation on $A$. More generally, a higher derivation on $A$ is a collection $D=$ $\left\{D^{i}\right\}$ of $\mathbf{k}$-linear maps on $A$ such that $D^{0}$ is the identity and the above Leibniz rule holds. The notion of higher derivations is due to H. Hasse and F.K. Schmidt [HS]. When the characteristic of $A$ is zero, each $D^{i}$ is determined by $D^{1}$, which is a locally nilpotent derivation on $A$. In this case, $\varphi=\exp \left(U D^{1}\right)=\sum_{i} \frac{1}{i !}\left(U D^{1}\right)^{i}$ and $A^{\varphi}$ is the kernel of $D^{1}$.

The above discussion of exponential maps, locally finite iterative higher derivations, and the AK invariant makes sense more generally for any (not necessarily commutative) ring. However, we will not need this generality.

Degree functions and related lemmas. Given an exponential map $\varphi: A \rightarrow$ $A[U]$ on a domain $A$ over $\mathbf{k}$, we can define the $\varphi$-degree of an element $a \in A$ by $\operatorname{deg}_{\varphi}(a)=\operatorname{deg}_{U}(\varphi(a))$ (where $\left.\operatorname{deg}_{U}(0)=-\infty\right)$. Note that $A^{\varphi}$ consists of all elements of $A$ with nonpositive $\varphi$-degree. The function $\operatorname{deg}_{\varphi}$ is a degree function on $A$, i.e. it satisfies these two properties for all $a, b \in A$ :

(i) $\operatorname{deg}_{\varphi}(a b)=\operatorname{deg}_{\varphi}(a)+\operatorname{deg}_{\varphi}(b)$.

(ii) $\operatorname{deg}_{\varphi}(a+b) \leq \max \left\{\operatorname{deg}_{\varphi}(a), \operatorname{deg}_{\varphi}(b)\right\}$.

We now collect some useful lemmas. The proofs are straightforward, and the reader can refer to the paper [CM] for those proofs which have been omitted.

Lemma 2.2. Let $\varphi$ be an exponential map on a domain $A$ over $\mathbf{k}$. Let $D=\left\{D^{i}\right\}$ be the locally finite iterative higher derivation associated to $\varphi$.

(a) If $a, b \in A$ such that $a b \in A^{\varphi} \backslash 0$, then $a, b \in A^{\varphi}$. In other words, $A^{\varphi}$ is factorially closed in $A$.

(b) $A^{\varphi}$ is algebraically closed in $A$.

(c) For each $a \in A, \operatorname{deg}_{\varphi}\left(D^{i}(a)\right) \leq \operatorname{deg}_{\varphi}(a)-i$. In particular, if $a \in A \backslash 0$ and $n=\operatorname{deg}_{\varphi}(a)$, then $D^{n}(a) \in A^{\varphi}$.

Proof. Refer to Lemma 2.1 of [CM].

Lemma 2.3. Let $\varphi$ be a nontrivial exponential map (i.e. not the standard inclusion) on a domain $A$ over $\mathbf{k}$ with $\operatorname{char}(\mathbf{k})=p \geq 0$. Let $x \in A$ with minimal positive $\varphi$-degree $n$.

(a) $D^{i}(x) \in A^{\varphi}$ for each $i \geq 1$. Moreover, $D^{i}(x)=0$ whenever $i \geq 1$ is not a power of $p$.

(b) If $a \in A \backslash 0$, then $n$ divides $\operatorname{deg}_{\varphi}(a)$. 
(c) Let $c=D^{n}(x)$. Then $A$ is a subalgebra of $A^{\varphi}\left[c^{-1}\right][x]$, where $A^{\varphi}\left[c^{-1}\right] \subseteq$ $\operatorname{Frac}\left(A^{\varphi}\right)$ is the localization of $A^{\varphi}$ at $c$.

(d) Let $\operatorname{trdeg}_{\mathbf{k}}$ denote transcendence degree over $\mathbf{k}$. If $\operatorname{trdeg}_{\mathbf{k}}(A)$ is finite, then $\operatorname{trdeg}_{\mathbf{k}}\left(A^{\varphi}\right)=\operatorname{trdeg}_{\mathbf{k}}(A)-1$.

Proof. For parts (a) through (c), refer to Lemma 2.2 of [CM]. Part (d) is immediate from part (c), together with part (b) of Lemma 2.2 which states that $A^{\varphi}$ is algebraically closed in $A$.

Lemma 2.4. Let $A$ be a domain over $\mathbf{k}$ with $\operatorname{trdeg}_{\mathbf{k}}(A)=1$. Then $\operatorname{AK}(A)=\mathbf{k}$ if and only if $A \cong \mathbf{k}^{[1]}$. Otherwise, $\operatorname{AK}(A)=A$.

Proof. Refer to Lemma 2.3 of [CM].

Thus $\mathbf{k}^{[1]}$ is the only transcendence degree 1 domain over $\mathbf{k}$ which admits nontrivial exponential maps.

Lemma 2.5. Let $\varphi$ be an exponential map on a domain $A$ over $\mathbf{k}$. Extend $\varphi$ to a homomorphism $\varphi: \operatorname{Frac}(A) \rightarrow \operatorname{Frac}(A)(U)$ by $\varphi\left(a b^{-1}\right)=\varphi(a) \varphi(b)^{-1}$, and let $\operatorname{Frac}(A)^{\varphi}=\{f \in \operatorname{Frac}(A) \mid \varphi(f)=f\}$. Then $\operatorname{Frac}(A)^{\varphi}=\operatorname{Frac}\left(A^{\varphi}\right)$.

Proof. It is clear that $\operatorname{Frac}\left(A^{\varphi}\right) \subseteq \operatorname{Frac}(A)^{\varphi}$. Suppose that $a, b \in A \backslash 0$, such that $a b^{-1} \in \operatorname{Frac}(A)^{\varphi}$. Then

$$
a b^{-1}=\varphi\left(a b^{-1}\right)=\varphi(a) \varphi(b)^{-1}=\left(\sum_{i} D^{i}(a) U^{i}\right)\left(\sum_{i} D^{i}(b) U^{i}\right)^{-1},
$$

and so

$$
\sum_{i} a D^{i}(b) U^{i}=\sum_{i} b D^{i}(a) U^{i}
$$

Compare the leading coefficients to see that $a$ and $b$ have the same $\varphi$-degree, say $n$, and $a b^{-1}=D^{n}(a) D^{n}(b)^{-1} \in \operatorname{Frac}\left(A^{\varphi}\right)$.

Let $A$ be a domain over $\mathbf{k}$, and let $\varphi \in \operatorname{EXP}(A)$. Let $B$ be the domain over $\operatorname{Frac}\left(A^{\varphi}\right)$ obtained by localizing $A$ at the multiplicative set $A^{\varphi} \backslash 0$. By Lemma 2.5. $\varphi$ extends to a $\operatorname{Frac}\left(A^{\varphi}\right)$-homomorphism $\varphi: B \rightarrow B[U]$ which is an exponential map on $B$ with ring of invariants $\operatorname{Frac}\left(A^{\varphi}\right)$.

Weights. We often produce a degree function on a domain $A$ by assigning degree values to some specified generators. On a product of generators the degree is then defined by the above property (i) of degree functions. Each element of $A$ can be expressed as a summation of linearly independent terms, each of which is a product of generators. The degree of such an expression is then defined to be the highest degree occurring among the terms. This is the case with the usual degree functions on polynomials, which are defined by assigning values to the indeterminates. In this situation we say that the degree function is obtained by assigning weights to some generators.

Homogenization of an exponential map. Let $A$ be a domain over $\mathbf{k}$ with exponential map $\varphi: A \rightarrow A[U]$. We now describe how, under certain circumstances, to obtain from $\varphi$ an exponential map $\bar{\varphi}$ on a graded version of $A$. Let $\mathbf{Z}$ denote the integers. Suppose that $A$ has a $\mathbf{Z}$-filtration $\left\{A_{n}\right\}$. This means that $A$ is the union of linear subspaces $A_{n}$ with these properties:

(i) $A_{i} \subseteq A_{j}$ whenever $i \leq j$. 
(ii) $A_{i} \cdot A_{j} \subseteq A_{i+j}$ for all $i, j \in \mathbf{Z}$.

(iii) $\bigcap_{n \in \mathbf{Z}} A_{n}=0$.

Given $a \in A \backslash 0$ there exists $i \in \mathbf{Z}$ for which $a \in A_{i} \backslash A_{i-1}$. Write

$$
\bar{a}=a+A_{i-1} \in A_{i} / A_{i-1},
$$

the top part of a. We can construct a graded $\mathbf{k}$-algebra

$$
\operatorname{gr}(A)=\bigoplus_{n \in \mathbf{Z}} A_{n} / A_{n-1}
$$

(Addition on $\operatorname{gr}(A)$ is given by its vector space structure. Given $\bar{a}=a+A_{i-1}$ and $\bar{b}=b+A_{j-1}$, multiplication is defined by $\bar{a} \bar{b}=a b+A_{i+j-1}$ and extended to all of $\operatorname{gr}(A)$ by the distributive law.)

On the filtration $\left\{A_{n}\right\}$ let us additionally assume that

$$
\left(A_{i} \backslash A_{i-1}\right) \cdot\left(A_{j} \backslash A_{j-1}\right) \subseteq A_{i+j} \backslash A_{i+j-1}
$$

for all $i, j \in \mathbf{Z}$. This will be the case if the filtration is induced by a degree function. Note then that $\bar{a} \bar{b}=\overline{a b}$ and that $\operatorname{gr}(A)$ is a domain.

Let grdeg be the degree function induced by the grading on $\operatorname{gr}(A)$. By assigning a weight to $\operatorname{grdeg}(U)$ for an indeterminate $U$-call the weight $\operatorname{grdeg}(U)$-we can extend the grading on $\operatorname{gr}(A)$ to $\operatorname{gr}(A)[U]$. Given the exponential map $\varphi: A \rightarrow A[U]$ on $A$, the goal is to obtain an exponential map $\bar{\varphi}: \operatorname{gr}(A) \rightarrow \operatorname{gr}(A)[U]$ on $\operatorname{gr}(A)$ by choosing the appropriate weight of $U$.

For $a \in A$, let $\operatorname{grdeg}(a) \operatorname{denote} \operatorname{grdeg}(\bar{a})$. Since $\operatorname{grdeg}(\bar{a})=i$ if and only if $a \in A_{i} \backslash A_{i-1}$, grdeg can also be viewed as a degree function on $A$ and on $A[U]$ once the value of $\operatorname{grdeg}(U)$ is determined.

Suppose that $\chi$ is a set of generators for $A$ over $\mathbf{k}$ with the following property: Given $i \in \mathbf{Z}$ and $a \in A_{i}$, we can write $a$ as a summation of monomials in elements of $\chi$ such that each monomial belongs to $A_{i}$. This is not an unreasonable property. It merely asserts some homogeneity on the generating set $\chi$. Under this assumption, $\operatorname{gr}(A)$ is generated by the top parts of the elements of $\chi$. Define

$$
\operatorname{grdeg}(U)=\min \left\{\frac{\operatorname{grdeg}(x)-\operatorname{grdeg}\left(D^{i}(x)\right)}{i} \mid x \in \chi, i \in \mathbf{Z}^{+}\right\} .
$$

Let us now assume that $\operatorname{grdeg}(U)$ does exist, i.e. is a rational number. (This will indeed occur whenever $\chi$ is a finite set, as will be the case with the Russell hypersurface of interest.) By our choice of $\operatorname{grdeg}(U)$ we have $\operatorname{grdeg}\left(D^{n}(x) U^{n}\right) \leq$ $\operatorname{grdeg}(x)$ for each $x \in \chi$ and each natural number $n$, and this inequality is sharp. By our homogeneity assumption on $\chi$ it follows that $\operatorname{grdeg}\left(D^{n}(a) U^{n}\right) \leq \operatorname{grdeg}(a)$ for all $a \in A$ and all natural numbers $n$. Given $a \in A$, let

$$
S(a)=\left\{n \mid \operatorname{grdeg}\left(D^{n}(a) U^{n}\right)=\operatorname{grdeg}(a)\right\}
$$

and define

$$
\bar{\varphi}(\bar{a})=\sum_{n \in S(a)} \overline{D^{n}(a)} U^{n}
$$

Extend this linearly to define $\bar{\varphi}: \operatorname{gr}(A) \rightarrow \operatorname{gr}(A)[U]$, the homogenization or top part of $\varphi$. This map is in fact an exponential map on $\operatorname{gr}(A)$. (See DHM for explicit demonstration of the exponential properties.) Let $\overline{A^{\varphi}}$ denote the domain generated by the top parts of all elements in $A^{\varphi}$. The end result is the following theorem. 
Theorem 2.6 (H. Derksen, O. Hadas, L. Makar-Limanov [DHM]). Let $A$ be a domain over $\mathbf{k}$ with $\mathbf{Z}$-filtration $\left\{A_{n}\right\}$ such that $\left(A_{i} \backslash A_{i-1}\right) \cdot\left(A_{j} \backslash A_{j-1}\right) \subseteq A_{i+j} \backslash$ $A_{i+j-1}$ for all $i, j \in \mathbf{Z}$. Let $\varphi$ be a nontrivial exponential map on A. Assume that $\operatorname{grdeg}(U)$ exists as defined above. Then $\bar{\varphi}$ as defined above is a nontrivial exponential map on $\operatorname{gr}(A)$. Moreover, $\overline{A^{\varphi}}$ is contained in $\operatorname{gr}(A)^{\bar{\varphi}}$.

An important special case of homogenization is when $A$ itself is graded. Then we can filter $A$ so that $\operatorname{gr}(A)$ is canonically isomorphic to $A$, and we can choose $\chi$ to be a set of homogeneous generators of $A$. In this case the top part of $\varphi$ is a nontrivial exponential map on $A$ (assuming $\operatorname{grdeg}(U)$ exists).

Example 2.7. Let $A=\mathbf{k}[X, Y]$, where $\operatorname{char}(\mathbf{k})=p$, prime. Define $\varphi \in \operatorname{EXP}(A)$ by $\varphi(X)=X$ and $\varphi(Y)=Y+U+X U^{p}$. We can grade $A$ by assigning weights $\operatorname{grdeg}(X)=\alpha$ and $\operatorname{grdeg}(Y)=\beta$ (with $\operatorname{grdeg}(\lambda)=0$ for all $\lambda \in \mathbf{k}^{*}$, and $\operatorname{grdeg}(0)=$ $-\infty)$. Since $\operatorname{grdeg}\left(D^{i}(X)\right)=-\infty$ for all $i \geq 1, X$ will not contribute to the value of $\operatorname{grdeg}(U)$. Therefore,

$$
\begin{aligned}
\operatorname{grdeg}(U) & =\min \left\{\frac{\operatorname{grdeg}(Y)-\operatorname{grdeg}(1)}{1}, \frac{\operatorname{grdeg}(Y)-\operatorname{grdeg}(X)}{p}\right\} \\
& =\min \left\{\beta, \frac{\beta-\alpha}{p}\right\} .
\end{aligned}
$$

In any case, $\bar{\varphi}(X)=X$. If $\beta<\frac{1}{p}(\beta-\alpha)$, then $\operatorname{grdeg}(U)=\beta$ and $\bar{\varphi}(Y)=Y+U$. If $\beta=\frac{1}{p}(\beta-\alpha)$, then $\operatorname{grdeg}(U)=\beta$ and $\bar{\varphi}(Y)=\varphi(Y)$. If $\beta>\frac{1}{p}(\beta-\alpha)$, then $\operatorname{grdeg}(U)=\frac{1}{p}(\beta-\alpha)$ and $\bar{\varphi}(Y)=Y+X U^{p}$.

\section{The Russell hypersurface}

Let $R=\mathbf{k}[X, Y, Z, T] /\left(X+X^{2} Y+Z^{2}+T^{3}\right)$. If we wish to emphasize a choice of $\mathbf{k}$, we write $R=R_{\mathbf{k}}$. Let $x, y, z, t \in R$ denote the cosets of $X, Y, Z, T$, respectively. We shall prove

Theorem 3.1. $\operatorname{AK}(R)=\mathbf{k}[x]$ for any $\mathbf{k}$.

Since $\operatorname{AK}\left(\mathbf{k}^{[3]}\right)=\mathbf{k}$, we have

Corollary 3.2. $R \varsubsetneqq \mathbf{k}^{[3]}$ for any $\mathbf{k}$.

To prove Theorem 3.1, let us start with

Lemma 3.3. $x \in \operatorname{AK}(R)$.

Proof. If we prove the lemma under the assumption that $\mathbf{k}$ is algebraically closed, then the result will follow for any field. For suppose that $\mathbf{F}$ is a field with algebraic closure $\mathbf{k}$. Then $R_{\mathbf{F}} \otimes_{\mathbf{F}} \mathbf{k}=R_{\mathbf{k}}$. So any $\varphi \in \operatorname{EXP}\left(R_{\mathbf{F}}\right)$ extends to $\varphi^{\prime} \in \operatorname{EXP}\left(R_{\mathbf{k}}\right)$ in a way which introduces only the elements of $\mathbf{k}$ as new invariants. If we know that $x$ is an invariant of $\varphi^{\prime}$, then $x$ must also be an invariant of $\varphi$. So let us now assume that $\mathbf{k}$ is algebraically closed.

Suppose that $\varphi: R \rightarrow R[U]$ is a nontrivial exponential map on $R$. We want to show that $x \in R^{\varphi}$. Let us consider $R$ as a subalgebra of $\mathbf{k}\left[x, x^{-1}, z, t\right]$ with

$$
y=-x^{-2}\left(x+z^{2}+t^{3}\right) .
$$

Introduce a degree function $w_{1}$ on $\mathbf{k}\left[x, x^{-1}, z, t\right]$ by assigning weights to the generators as follows: $w_{1}(x)=-1, w_{1}(z)=0$, and $w_{1}(t)=0$ (with $w_{1}(\lambda)=0$ for all 
$\lambda \in \mathbf{k}^{*}$ and $\left.w_{1}(0)=-\infty\right)$. Note that $w_{1}(y)=2$. This degree function induces a Z-filtration $\left\{R_{i}\right\}$ on $R$, where $R_{i}$ consists of all $r \in R$ with $w_{1}(r) \leq i$. Passing to top parts, $\bar{y}=-\bar{x}^{-2}\left(\bar{z}^{2}+\bar{t}^{3}\right)$. So the corresponding graded domain $\operatorname{gr}(R)$ is generated by $\bar{x}, \bar{y}, \bar{z}, \bar{t}$ and subject to the relation $\bar{x}^{2} \bar{y}+\bar{z}^{2}+\bar{t}^{3}=0$. (There cannot be any other relations because $\bar{x}, \bar{z}$, and $\bar{t}$ are algebraically independent.) Let us write $x, y, z, t$ in place of $\bar{x}, \bar{y}, \bar{z}, \bar{t}$, respectively. Then

$$
\operatorname{gr}(R)=\mathbf{k}[x, y, z, t] /\left(x^{2} y+z^{2}+t^{3}\right) .
$$

For a first step we show

Sublemma 3.4. $R^{\varphi} \subseteq \mathbf{k}[x, z, t]$.

Proof. Suppose for the sake of contradiction that $f \in R^{\varphi}$ and $f \notin \mathbf{k}[x, z, t]$. It is clear that the value $\operatorname{grdeg}(U)$ as defined by formula (因) exists for our filtration, taking $\chi=\{x, y, z, t\}$. By Theorem 2.6, $\varphi$ induces a nontrivial exponential map $\bar{\varphi}$ on $\operatorname{gr}(R)$ with $\bar{f} \in \operatorname{gr}(R)^{\bar{\varphi}}$. We can write

$$
\bar{f}=x^{a} y^{b} g(z, t)
$$

for some natural numbers $a$ and $b$ and some polynomial $g(z, t) \in \mathbf{k}[z, t]$. By our assumption on $f$, we know $b$ must be positive. We can assume that $a=0$ or $a=1$, since a factor $x^{2} y$ of $\bar{f}$ can be absorbed into $g(z, t)$ by the relation on $\operatorname{gr}(R)$. Let us even assume that $a=0$. For if $a=1$, then we can replace $f$ by $f^{2}$ in our arguments. Thus we have an element $\bar{f}=y^{b} g(z, t)$ in $\operatorname{gr}(R)^{\bar{\varphi}}$. Also, $\operatorname{trdeg}_{\mathbf{k}}\left(R^{\varphi}\right)=2$ by part (d) of Lemma 2.3. So we can assume that $g(z, t)$ is not a constant polynomial by replacing $f$ if necessary with another element of $R^{\varphi}$.

By part (a) of Lemma 2.2, $\operatorname{gr}(R)^{\bar{\varphi}}$ is factorially closed. So both $y$ and $g(z, t)$ belong to $\operatorname{gr}(R)^{\bar{\varphi}}$. We introduce a new grading on $\operatorname{gr}(R)$ by the weights $w_{2}(x)=6$, $w_{2}(y)=-6, w_{2}(z)=3$, and $w_{2}(t)=2$. This gives us a graded domain $\operatorname{gr}(\operatorname{gr}(R))$ which is naturally isomorphic to $\operatorname{gr}(R)$. Let us write $\operatorname{gr}(R)$ in place of $\operatorname{gr}(\operatorname{gr}(R))$, and let us continue to write $x, y, z, t$ in place of $\bar{x}, \bar{y}, \bar{z}, \bar{t}$. The effect of imposing $w_{2}$ on $\operatorname{gr}(R)$ is to refine the top parts that were obtained via $w_{1}$, so that on the elements $x, y, z, t$ there are two weights: the primary weights given by $w_{1}$ and the secondary weights given by $w_{2}$. By Theorem 2.6 we obtain a new nontrivial exponential map $\overline{\bar{\varphi}}$ on $\operatorname{gr}(R)$, a "refinement" of $\bar{\varphi}$. But let us avoid this double-bar notation and use $\bar{\varphi}$ to denote the homogenization of $\varphi$ under the primary and secondary weights $w_{1}$ and $w_{2}$. Under this combination of weights $\bar{f}$ is now $w_{2}$-homogeneous, so that $g(z, t)$ factors as

$$
g(z, t)=\lambda z^{n} t^{m} \prod_{i}\left(z^{2}+\mu_{i} t^{3}\right)
$$

for some natural numbers $n$ and $m$ and for some $\lambda, \mu_{i} \in \mathbf{k}^{*}$. Note that each factor of $g(z, t)$ belongs to $\operatorname{gr}(R)^{\bar{\varphi}}$.

The next step is to show that neither $z$ nor $t$ can be $\bar{\varphi}$-invariant. Our final contradiction will occur when we cannot reconcile this observation with the above factorization of $g(z, t)$. Suppose that $z \in \operatorname{gr}(R)^{\bar{\varphi}}$. Recalling that $y \in \operatorname{gr}(R)^{\bar{\varphi}}$ as well, we have $\mathbf{k}[y, z] \subseteq \operatorname{gr}(R)^{\bar{\varphi}}$. In fact, since $\operatorname{gr}(R)^{\bar{\varphi}}$ is algebraically closed in $\operatorname{gr}(R)$ with $\operatorname{trdeg}_{\mathbf{k}}\left(\operatorname{gr}(R)^{\bar{\varphi}}\right)=2$, we have $\operatorname{gr}(R)^{\bar{\varphi}}=\mathbf{k}[y, z]$. By virtue of the remark following Lemma 2.5. $\bar{\varphi}$ induces a nontrivial exponential map on the domain

$$
\mathbf{k}(y, z)[x, t] /\left(x^{2} y+z^{2}+t^{3}\right) .
$$


But this domain has transcendence degree 1 over $\mathbf{k}(y, z)$ and is not isomorphic to $\mathbf{k}(y, z)^{[1]}$. This contradicts Lemma 2.4. Thus $z \notin \operatorname{gr}(R)^{\bar{\varphi}}$. Suppose now that $t \in \operatorname{gr}(R)^{\bar{\varphi}}$. In this case $\operatorname{gr}(R)^{\bar{\varphi}}=\mathbf{k}[y, t]$, and just as with $z$ we can obtain a nontrivial exponential map on the domain

$$
\mathbf{k}(y, t)[x, z] /\left(x^{2} y+z^{2}+t^{3}\right) .
$$

Again, this domain has transcendence degree 1 over $\mathbf{k}(y, t)$ but is not isomorphic to $\mathbf{k}(y, t)^{[1]}$, contradicting Lemma [2.4. So $t \notin \operatorname{gr}(R)^{\bar{\varphi}}$ as well. Since $\operatorname{gr}(R)^{\bar{\varphi}}$ is factorially closed, $n=0$ and $m=0$ in the above factorization of $g(z, t)$.

It remains to consider the factors of $g(z, t)$ of the form $z^{2}+\mu_{i} t^{3}$. Ignoring multiplicity, there can only be one such factor. Indeed, given two factors of this type, their difference belongs to $\operatorname{gr}(R)^{\bar{\varphi}}$ from which we conclude that both $z^{2}$ and $t^{3}$ belong to $\operatorname{gr}(R)^{\bar{\varphi}}$, which we have just shown to be impossible. Also, $z^{2}+t^{3}$ cannot be a factor of $g(z, t)$, since otherwise we would have $x^{2} y=-\left(z^{2}+t^{3}\right) \in \operatorname{gr}(R)^{\bar{\varphi}}$, which in turn implies that $\operatorname{gr}(R)^{\bar{\varphi}}=\operatorname{gr}(R)$, contradicting the nontriviality of $\bar{\varphi}$. We can therefore write $g(z, t)$ as

$$
g(z, t)=\lambda\left(z^{2}+\mu t^{3}\right)^{k}
$$

for some positive integer $k$ and some $\lambda, \mu \in \mathbf{k}^{*}$ with $\mu \neq 1$. We will use the same trick that worked for $z$ and $t$. Let $S$ be the domain which results from localizing $\operatorname{gr}(R)$ at $\operatorname{gr}(R)^{\bar{\varphi}} \backslash 0$. Recall that $y$ and $z^{2}+\mu t^{3}$ belong to $\operatorname{gr}(R)^{\bar{\varphi}}$. Note that we can rewrite $x^{2} y+z^{2}+t^{3}=0$ as the relation

$$
x^{2} y+(1-\mu) t^{3}+\left(z^{2}+\mu t^{3}\right)=0
$$

over $\operatorname{Frac}\left(\operatorname{gr}(R)^{\bar{\varphi}}\right)$. From this we can see that $S$ has transcendence degree 1 over $\operatorname{Frac}\left(\operatorname{gr}(R)^{\bar{\varphi}}\right)$ but is not isomorphic to $\operatorname{Frac}\left(\operatorname{gr}(R)^{\bar{\varphi}}\right)^{[1]}$. We can extend $\bar{\varphi}$ to a nontrivial exponential map on $S$ over $\operatorname{Frac}\left(\operatorname{gr}(R)^{\bar{\varphi}}\right)$ in the manner described after Lemma 2.5. This once again contradicts Lemma 2.4. We have now exhausted all possibilities. To avoid a contradiction, we must have $f \in \mathbf{k}[x, z, t]$. So $R^{\varphi} \subseteq$ $\mathbf{k}[x, z, t]$.

Continuing with the proof of Lemma 3.3, we are now in a position to show that $x \in R^{\varphi}$. Suppose that it is not the case. If $f \in R^{\varphi} \subseteq \mathbf{k}[x, z, t]$, write $f=x f_{1}(x, z, t)+f_{2}(z, t)$. Then $f_{2} \neq 0$ since $x \notin R^{\varphi}$. Again consider $\operatorname{gr}(R)$ given by $w_{1}$. Now $w_{1}\left(x f_{1}(x, z, t)\right)$ is negative, while $w_{1}\left(f_{2}(z, t)\right)=0$, and so $\bar{f}=$ $f_{2}(z, t) \in \operatorname{gr}(R)^{\bar{\varphi}}$. Let $g \in R^{\varphi}$ be algebraically independent with $f$ over k. (Recall that $\operatorname{trdeg}_{\mathbf{k}}\left(R^{\varphi}\right)=2$ by part (d) of Lemma 2.3.) We write $g=x g_{1}(x, z, t)+g_{2}(z, t)$, where $0 \neq g_{2}(z, t)=\bar{g}$. Suppose that $f_{2}$ and $g_{2}$ are algebraically dependent over $\mathbf{k}$, say $P\left(f_{2}, g_{2}\right)=0$. Then $P(f, g)$ is a nonzero element of $R^{\varphi}$, but $P(f, g)$ is divisible by $x$. This implies that $x \in R^{\varphi}$, contrary to our assumption. Hence it must be that $f_{2}$ and $g_{2}$ are algebraically independent over $\mathbf{k}$. Thus $\operatorname{gr}(R)^{\bar{\varphi}}$ contains two algebraically independent elements of $\mathbf{k}[z, t]$. Since $\operatorname{gr}(R)^{\bar{\varphi}}$ is algebraically closed in $\operatorname{gr}(R)$ (part (b) of Lemma 2.2), we deduce that $\operatorname{gr}(R)^{\bar{\varphi}}=\mathbf{k}[z, t]$. Now $x^{2} y=-z^{2}-t^{3} \in \operatorname{gr}(R)^{\bar{\varphi}}$, and this implies that $x, y \in \operatorname{gr}(R)^{\bar{\varphi}}$. But then $\bar{\varphi}$ is trivial. This contradicts our assumption that $x \notin R^{\varphi}$. So $x \in R^{\varphi}$ for every $\varphi \in \operatorname{EXP}(R)$, and the lemma is finally proved.

Proof of Theorem 3.1. We know that $\mathbf{k}[x] \subseteq \mathrm{AK}(R)$. To show the reverse containment, we consider two maps $\varphi_{1}$ and $\varphi_{2}$ which one easily verifies to be exponential 
maps on $R$. Define $\varphi_{1}: R \rightarrow R[U]$ by

$$
\begin{aligned}
& \varphi_{1}(x)=x, \\
& \varphi_{1}(y)=y+2 z U-x^{2} U^{2}, \\
& \varphi_{1}(z)=z-x^{2} U, \\
& \varphi_{1}(t)=t .
\end{aligned}
$$

The ring of $\varphi_{1}$-invariants is $\mathbf{k}[x, t]$. Define $\varphi_{2}: R \rightarrow R[U]$ by

$$
\begin{aligned}
& \varphi_{2}(x)=x, \\
& \varphi_{2}(y)=y+3 t^{2} U-3 x^{2} t U^{2}+x^{4} U^{3}, \\
& \varphi_{2}(z)=z, \\
& \varphi_{2}(t)=t-x^{2} U .
\end{aligned}
$$

The ring of $\varphi_{2}$-invariants is $\mathbf{k}[x, z]$. So $\operatorname{AK}(R)$ is contained in the intersection of these two rings, that being $\mathbf{k}[x]$.

As a final remark, L. Makar-Limanov [M2] has recently taken a simplified approach (again very similar to the proof given here) to showing that $\operatorname{AK}\left(R_{\mathbf{C}}\right)=\mathbf{C}[x]$. This proof uses the following fact.

Lemma 3.5 (see [M2]). Let $A$ be a domain with characteristic zero. Let $n$ and $m$ be natural numbers both at least 2. Let $\varphi \in \operatorname{EXP}(A)$ and $c_{1}, c_{2} \in A^{\varphi} \backslash 0$. If $a, b \in A$ such that $c_{1} a^{n}+c_{2} b^{m} \in A^{\varphi} \backslash 0$, then $a, b \in A^{\varphi}$.

In fact, this lemma is still true when $A$ has prime characteristic $p$, under the additional necessary hypothesis that neither $n$ nor $m$ are powers of $p[\mathrm{C}$. This lemma can then replace the many times that we used Lemma 2.5 to contradict Lemma 2.4. But because of the extra assumption needed on Lemma 3.5, this method fails when the characteristic of $R$ is 2 or 3 .

\section{REFERENCES}

[BM] T. Bandman and L. Makar-Limanov, Affine surfaces with $A K(S)=\mathbf{C}$, Michigan Math. J. 49(2001), 567-582. MR1872757 (2003a:14094)

[C] A. Crachiola, On the AK invariant of certain domains, Ph.D. thesis, Wayne State University, 2004.

[CM] A. Crachiola and L. Makar-Limanov, On the rigidity of small domains, J. Algebra 284 (2005), no. 1, 1-12. MR2115001

[D] H. Derksen, More on the hypersurface $x+x^{2} y+z^{2}+t^{3}=0$ in $\mathbf{C}^{4}$, preprint, 1995, 4 pages.

[DHM] H. Derksen, O. Hadas, and L. Makar-Limanov, Newton polytopes of invariants of additive group actions, J. Pure Appl. Algebra 156(2001), 187-197. MR.1808822 (2002g:14068)

[Du] A. Dubouloz, Generalized Danielewski surfaces, preprint, 2004, 24 pages.

[E] A. van den Essen, Polynomial automorphisms and the Jacobian conjecture, Progr. Math., Vol. 190, Birkhäuser Verlag, Basel, 2000. MR1790619(2001j:14082)

[F] T. Fujita, On Zariski problem, Proc. Japan Acad. Ser. A Math. Sci. 55(1979), 106-110. MR0531454 (80j:14029)

[HS] H. Hasse and F.K. Schmidt, Noch eine Bergründung der Theorie der höheren Differentialquotienten in einem algebraischen Functionenkörper einer Unbestimmten, J. Reine Angew. Math. 177(1937), 215-237.

[KKMR] S. Kaliman, M. Koras, L. Makar-Limanov, and P. Russell, $\mathbf{C}^{*}$-actions on $\mathbf{C}^{3}$ are linearizable, Electron. Res. Announc. Amer. Math. Soc. 3(1997), 63-71. MR1464577 (98i:14046) 
[KR] M. Koras and P. Russell, Contractible threefolds and $\mathbf{C}^{*}$-actions on $\mathbf{C}^{3}$, J. Algebraic Geometry 6(1997), no. 4, 671-695. MR1487230 (99e:14057)

[M1] L. Makar-Limanov, On the hypersurface $x+x^{2} y+z^{2}+t^{3}=0$ in $\mathbf{C}^{4}$, or a $\mathbf{C}^{3}$-like threefold which is not $\mathbf{C}^{3}$, Israel J. Math. 96(1996), 419-429. MR.1433698 (98a:14052)

[M2] L. Makar-Limanov, Again $x+x^{2} y+z^{2}+t^{3}=0$, Contemp. Math., 369, Amer. Math. Soc., 2005. MR2126661

[Mi] M. Miyanishi, $G_{a}$-action of the affine plane, Nagoya Math. J. 41(1971), 97-100. MR0281719(43:7434)

[MS] M. Miyanishi and T. Sugie, Affine surfaces containing cylinderlike open sets, J. Math. Kyoto Univ. 20(1980), 11-42. MR0564667 (81h:14020)

[R] R. Rentschler, Opérations du groupe additif sur le plane affine, C.R. Acad. Sci. Paris 267(1968), 384-387. MR0232770 (38:1093)

[Ru] P. Russell, On affine-ruled rational surfaces, Math. Ann. 255(1981), 287-302. MR0615851 (82h:14024)

Department of Mathematics and Computer Science, Loyola University, New OrLEANS, LOUISIANA 70118

Current address: Department of Mathematical Sciences, Saginaw Valley State University, 7400 Bay Road, University Center, Michigan 48710-0001

E-mail address: crachiola@member.ams.org 